\title{
EDUCAÇÃO E ECOLOGIA PROFUNDA: REFLEXÕES SOBRE OS POTENCIAIS PEDAGÓGICOS DA HORTA ESCOLAR
}

\author{
Aline Schú ${ }^{1}$ \\ Cláudia Petry ${ }^{2}$ \\ Ivan Penteado Dourado ${ }^{3}$ \\ Janine Fleith de Medeiros ${ }^{4}$ \\ Jaime Martinez ${ }^{5}$
}

Resumo: O cultivo da terra pode proporcionar a sensibilização ambiental, o reconhecimento do valor intrínseco da natureza e promover a percepção de que a humanidade é parte da "teia da vida". Nesse sentido, este estudo, de caráter bibliográfico narrativo, tem como objetivo abordar a horta escolar como ferramenta pedagógica dentro do paradigma da ecologia profunda. A partir da pesquisa realizada sobre o tema, os achados literários encontrados somam um total de 75 artigos publicados, dentre os quais, 63 abordam a horta escolar sob uma perspectiva reducionista e produtivista e somente 12 estudos a relacionam a aspectos mais abrangentes, direcionados à ecologia profunda. São poucas as investigações que mencionam a horta escolar como instrumento metodológico educativo dentro da perspectiva salientada por este estudo.

Palavras Chave: Educação Ambiental; Pátio Escolar Comestível; Valor Intrínseco da Natureza.

Abstract: The cultivation of land can provide environmental awareness, recognition of the intrinsic value of nature and promote the perception that humanity is part of the "web of life". This narrative bibliographic study addresses the school garden as an educational tool within the paradigm of deep ecology. In the literature there is a total of 75 published articles, among which, 63 approach the school garden from a reductionist and productivist perspective and only 12 studies relate it to more comprehensive aspects, directed to aspects of deep ecology. There are few studies that mention the school garden as an educational methodological tool within the perspective of deep ecology.

Keywords: Edible School Yard; Intrinsic Value of Nature; Environmental Education. 


\section{Introdução}

A crise ambiental planetária origina-se das ações antrópicas sobre os ecossistemas e o clima. As escolhas humanas nas áreas econômica, social e ambiental ocasionaram pobreza, desigualdade social e a superexploração dos bens naturais.

O desrespeito aos ciclos naturais e o consumismo em excesso estão levando seres à extinção e comprometendo a funcionalidade dos ecossistemas. Ceballos et al. (2015) consideram que a Terra esteja passando pela sexta extinção em massa, e, mesmo com estudos insuficientes sobre o número de espécies existentes que foram extintas ao longo do tempo, há um consenso de que esse dado seja alto e esteja em constante crescimento.

Capra (1996) alerta que a instabilidade ambiental tem origem em uma crise de percepção na qual a realidade não é vislumbrada, isso porque boa parte dos humanos partilha de uma visão de mundo ultrapassada. Diante disso, para enfrentar essa crise, é necessária uma mudança significativa das percepções, pensamentos e valores.

Essa visão obsoleta manifesta-se na percepção distorcida de que a natureza é algo separado do ser humano e que possui somente valor utilitário (ou instrumental). Quando o ser humano é tomado como centro de tudo, privilegia-se uma perspectiva antropocêntrica. Com isso, a noção de interdependência entre os seres e a compreensão da complexa rede de interações que os unem ao meio são prejudicadas.

Existe uma ampla literatura que aborda o antropocentrismo como o pivô da crise ecológica. A fragmentação entre sujeito e objeto e Natureza e Cultura é uma das principais causas dos problemas ambientais (GRÜN, 2005). Essa cisão traduz a separação sujeito-objeto, característica importante do representacionismo. Tal concepção almeja que continuemos acreditando que somos algo separado do mundo e que ele existe independentemente de nossa experiência (MARIOTTI, 2001).

Como alternativa a essa concepção, estabelecem-se outros paradigmas, como a ecologia profunda. De acordo com Goldim (1998), o conceito do termo foi proposto pelo filósofo Arne Naess, em 1973. O intuito era dar uma resposta a esta visão predominante da época, antropocêntrica e representacionista, sobre a utilização dos bens naturais.

Arne Naess distinguiu "ecologia rasa" do que nomeou de "ecologia profunda". Adotou-se, neste artigo, com o objetivo de diferenciar esses termos, os conceitos estabelecidos por Capra (1996). A ecologia rasa é antropocêntrica e trata os humanos como superiores ou algo à parte da natureza, os trata como a fonte de todos os valores. A natureza, dentro dessa perspectiva, possui apenas valor instrumental. $\mathrm{Na}$ ecologia profunda, por outro lado, todos os seres são considerados interdependentes no ambiente natural. Existe uma rede de fenômenos interconectados e não uma coleção de objetos isolados. Assim, esse paradigma reconhece o valor intrínseco de todos os seres e trata o ser 
humano como parte integrante da teia da vida. Em caráter mais profundo, é uma percepção espiritual ou religiosa, pois o indivíduo possui o sentimento de pertinência e de conexão com o cosmos como um todo (CAPRA, 1996).

Assim, a ecologia profunda é sinônima da visão holística que idealiza o mundo em conexidade e não fragmentado. Essa percepção admite os fenômenos, os indivíduos e as sociedades como interdependentes e em imersão nos processos cíclicos da natureza dos quais são dependentes (CAPRA, 1996).

Nesse sentido, é preciso uma mudança nas formas de ver, ler e entender o mundo. Conforme Capra (2006, p. 14), três fenômenos devem ser considerados para entender a complexidade da vida: compreender que ela se organiza na forma de rede ou teia, que a matéria passa por ciclos nessa teia e que todos os processos são sustentados pelo fluxo contínuo de energia. Esses fenômenos são interiorizados pelas crianças na vivência cotidiana, por meio de experiências diretas com o mundo natural.

Portanto, o referido paradigma exige uma mudança nos processos de ensino e aprendizagem e em como cada ser humano se relaciona consigo, com os outros e com o planeta. A construção do conhecimento nas escolas é, em grande parte, feita dentro dos componentes curriculares, que podem apresentar dificuldades para conversar entre si. A exigência do cumprimento dos conteúdos programáticos pode atravancar discussões ambientais mais profundas. Conforme Lovatto et al. (2011, p. 123), "a civilização parece permanecer cega diante da ameaça à sua própria existência e continua gerando e educando crianças doutrinadas sob os mesmos valores".

O reconhecimento da centralidade da dimensão ambiental na educação brasileira emerge como Projeto de Lei por meio da Política Nacional de Educação Ambiental (PNEA), estabelecida pela Lei no 9.795, de 27 de abril de 1999, e regulamentada pelo Decreto no 4.281/2002, que dispõe e institui "A Educação Ambiental, como componente essencial e permanente da educação nacional, devendo estar presente, de forma articulada, em todos os níveis e modalidades do processo educativo, em caráter formal e não formal" (BRASIL, 2002, art. 2). Essa regulamentação passa a amparar, nos currículos escolares, o disposto da Constituição Federal (CF) de 1988, inciso VI do $\S 1^{\circ}$ do artigo 225, que determina que o Poder Público deve "promover a Educação Ambiental em todos os níveis de ensino e a conscientização pública para a preservação do meio ambiente" (BRASIL, 1998, art. 225).

No mundo, essa luta que se consolida historicamente na década de 1960, mas que no campo educacional recebe o devido reconhecimento pelos currículos brasileiros apenas no final da década de 1990, aponta para a recente inclusão da Consciência Ambiental nos currículos escolares, articulada, inclusive, pelo Sistema Nacional de Educação Ambiental (Sisnea), como tema transversal em todos os níveis de educação formal e não formal no país. 
Desde então, os profissionais da educação podem trabalhar a ecologia de maneira profunda, por meio de metodologias de ensino diferenciadas ou por meio da realização de projetos, como de criação de hortas escolares. O cultivo da terra pode proporcionar a sensibilização e o reconhecimento de que a humanidade é parte da "teia da vida". Assim, são reestabelecidas as relações de cuidado e respeito com o meio e os seres que o integram, o que pode proporcionar momentos de educação integral processados nos âmbitos social, cultural, intelectual, cognitivo, físico, emocional, espiritual e simbólico.

Tendo isso em vista, este artigo trata-se de um estudo teórico baseado em coleta de dados a partir de pesquisa bibliográfica. O estudo foi realizado considerando duas bases de dados, Scopus e Web of Science, as quais foram escolhidas porque possuem alto número de periódicos indexados. Assim, apresenta um recorte temporal, parte de um mapeamento de pesquisas já realizadas sobre a horta escolar como ferramenta educativa, buscando, por meio desses estudos, dialogar com a perspectiva da ecologia profunda. Desta forma, propõe a seguinte problemática: pesquisas realizadas referentes à horta escolar podem estar sujeitas a uma visão reducionista e produtivista? Quais seriam os potenciais pedagógicos que as pesquisas selecionadas relacionam ao uso da horta escolar na perspectiva da ecologia profunda sob a ótica da construção de sensibilização ambiental?

\section{Método}

Considerando o objetivo desta investigação, foi realizada uma pesquisa exploratória em dados secundários. Estudos exploratórios têm como objetivo explorar ou fornecer informações e maior compreensão sobre um problema ou situação enfrentada pelo pesquisador (MALHOTRA, 2012).

Desse modo, conduzimos uma pesquisa bibliográfica com o intuito de investigar se a horta escolar poderia estar sujeita a uma visão reducionista/produtivista e para verificar quais seriam os potenciais pedagógicos do uso dessa ferramenta na perspectiva da ecologia profunda, sob a ótica da construção da sensibilização ambiental.

Para operacionalizar a pesquisa, optamos por utilizar o método narrativo. Segundo Gregory e Denniss (2018), a pesquisa narrativa propõe um estudo não sistemático e a análise da literatura disponível sobre um assunto específico de interesse. Contudo, como essa forma de revisão não fornece uma metodologia para a busca das referências, decidimos observar um roteiro de orientação, cujas etapas estão descritas na Tabela 1. 
Tabela 1: Descrição das etapas para a Revisão Bibliográfica Narrativa.

\begin{tabular}{l|l}
\hline \multicolumn{1}{c|}{ ETAPAS } & \multicolumn{1}{c}{ DESCRIÇÃO } \\
\hline $\begin{array}{l}\text { Fontes para busca de } \\
\text { informação }\end{array}$ & $\begin{array}{l}\text { A busca nos periódicos foi restrita aos estudos armazenados } \\
\text { nas bases de dados Web of Science e Scopus. }\end{array}$ \\
\hline $\begin{array}{l}\text { Operacionalização da } \\
\text { busca }\end{array}$ & $\begin{array}{l}\text { Nas duas bases escolhidas, a busca foi realizada por meio dos } \\
\text { descritores: "Edible school yard" e "School vegetable garden". } \\
\text { Delimitamos como recorte temporal os trabalhos publicados } \\
\text { entre 2014 e 2018. Como filtro, utilizamos os termos presentes } \\
\text { no título e/ou resumo e/ou palavras-chave. }\end{array}$ \\
\hline $\begin{array}{l}\text { Seleção dos artigos lidos } \\
\text { e analisados }\end{array}$ & $\begin{array}{l}\text { Após exclusão dos artigos repetidos, a seleção foi realizada } \\
\text { através de leitura dos resumos e análise da congruência entre } \\
\text { as pesquisas com os objetivos deste estudo. }\end{array}$ \\
\hline $\begin{array}{l}\text { Análise dos artigos } \\
\text { selecionados }\end{array}$ & $\begin{array}{l}\text { Após a leitura, tendo em mente os objetivos deste trabalho, foi } \\
\text { realizada a seleção de contribuições relevantes para esta } \\
\text { produção. Como o método de análise é de revisão narrativa, } \\
\text { justifica-se o uso de interpretação e análise crítica pessoal dos } \\
\text { pesquisadores. }\end{array}$ \\
\hline
\end{tabular}

Fonte: Autoria própria.

Na seção a seguir são apresentadas reflexões sobre educação, políticas públicas e ecologia profunda, para, em seguida, ser demonstrada a análise quantitativa e qualitativa realizada sobre os artigos selecionados nas bases de dados anteriormente referidas. É feito um contraponto entre as perspectivas produtivista/reducionista e ecologia profunda sobre a utilização da horta escolar como metodologia de ensino voltada ao desenvolvimento de valores sustentáveis.

\section{Aprendizagem por meio da horta escolar}

A Política Nacional de Educação Ambiental (Lei n.o 9.795/99) salienta que a Educação Ambiental não deve ser implantada como um componente curricular isolado, pois deve perpassar todos os demais componentes e os projetos escolares (BRASIL, 1999). Educar em um mundo em crise força uma ruptura com os modelos antropocêntricos aos quais os profissionais da educação foram e são expostos no decorrer de suas trajetórias formativas. Esse esforço é necessário para que consigam educar para a sustentabilidade e promover valores direcionados ao biocentrismo, onde todas as formas de vida têm relevância.

Segundo Sauvé (2005), um conjunto de dimensões metodológicas e teóricas entrelaçadas e complementares permeia as relações com o meio ambiente. A Educação Ambiental não pode se restringir a uma ou a outra dessas dimensões, já que corre o risco de se tornar incompleta e de distorcer a visão sobre o papel de cada um no mundo. 
Para Soria (2012), a ressignificação da ecologia acontece ao longo do tempo, o que explica porque existem tantas derivações colocadas como adjetivos depois do termo ecologia. A ecologia profunda pode ser vislumbrada como a "continuidade histórica da questão ecológica e que estabelece uma ligação relacional entre o interno e o externo do ser humano, que tenta ir além da ecologia como ciência comum para abrir caminho para questionamentos de ordem filosófica e espiritual" (p. 28).

Nesse sentido, a escola deve promover atividades e estimular 0 fortalecimento de valores que visem educar para a sustentabilidade. Contudo, é necessário reconhecer as limitações do conhecimento e das percepções construídas pelos humanos até a atualidade, bem como é necessário desenvolver novas abordagens de ensinar, aprender e de apreender o mundo. O valor intrínseco da natureza deve ser reconhecido e o seu valor instrumental minimizado. Conforme Grün, o problema epistemológico originado do antropocentrismo cartesiano é a fragmentação do "objeto", ou seja,

a fragmentação do objeto nos impede de ter uma visão complexa do meio ambiente em Educação Ambiental e Ética Ambiental. O antropocentrismo fica evidente na tentativa de Descartes de conferir autonomia à razão, que faz do mundo um objeto manejável. A unidade da razão (autonomia) se dá por meio da divisibilidade (fragmentação) do mundo físico (GRÜN, 2005, p. 46).

Conforme esse autor, o antropocentrismo cartesiano é "reducionista, fragmentário, sem vida e mecânico", por isso, ele sugere um modelo que seja "complexo, holístico, vivo e orgânico" (GRÜN, 2002, p. 63).

Para Leff (2002), a solução da crise ambiental global e planetária, não será realizada apenas por meio da gestão racional da natureza e dos riscos da mudança global. A crise nos questiona sobre o conhecimento do mundo, sobre o projeto epistemológico que busca a unidade, uniformidade e homogeneidade e que anuncia um futuro comum. Esse projeto nega o limite, o tempo, a história, a diferença, a diversidade e a outridade, e destaca que

a complexidade ambiental inaugura uma nova reflexão sobre a natureza do ser, do saber e do conhecer, sobre a hibridização de conhecimentos na interdisciplinaridade e transdisciplinaridade; sobre o diálogo dos saberes e a inserção da subjetividade, dos valores e interesses nas tomadas de decisão e nas estratégias de apropriação da natureza. Mas questiona também as formas pelas quais os valores permeiam o conhecimento do mundo, abrindo um espaço para o encontro entre o racional e o moral, entre a racionalidade formal e a racionalidade substantiva (LEFF, 2002, p. 195). 
Leff (2002, p. 192) afirma que a complexidade não pode ser reduzida ao "reflexo de uma realidade complexa no pensamento". A complexificação do mundo é o encontro do ser com a construção do pensamento complexo.

Aprender a complexidade ambiental implica um processo de desconstrução e reconstrução do pensamento; remete-nos às suas origens, a compreensão de suas causas; implica considerar os 'erros' da história que se enraizaram em certezas sobre o mundo com falsos fundamentos; descobrir e reavivar o ser da complexidade que foi 'esquecido' com o surgimento da cisão entre o ser e o ente (Platão), do sujeito e do objeto (Descartes), para aprender 0 mundo coisificando-o, objetivando-o, homogeneizando-o. Esta racionalidade dominante descobre a complexidade a partir de seus limites, a partir de sua negatividade, a partir da alienação e da incerteza do mundo economizado, arrastado por um processo incontrolável e insustentável de produção (LEFF, 2002, p. 192).

A sociedade contemporânea busca incessantemente o lucro. O modelo antropocêntrico de desenvolvimento tem infligido ao planeta os mais variados impactos ambientais. Dentre eles, podem ser citados os prejuízos aos processos cíclicos naturais, os danos à saúde, às teias alimentares e às relações entre os seres vivos. Foram criados organismos geneticamente modificados, há abuso no uso de agrotóxicos, os alimentos contêm excesso de aditivos químicos e conservantes e as florestas cederam espaço ao solo com extensas áreas voltadas à monocultura.

Não obstante, é pertinente debater nas escolas os temas ambientais. A Lei $\mathrm{n}^{\circ} 11.346$ estabelece o Sistema Nacional de Segurança Alimentar e Nutricional e salienta que o direito humano à alimentação adequada deve ser assegurado. O poder público, com a participação da sociedade civil organizada, deve formular e implementar políticas, planos, programas e ações que almejem a garantia desse direito (BRASIL, 2006).

O "Marco de Referência de Educação Alimentar e Nutricional para as Políticas Públicas" conceitua a Educação Alimentar e Nutricional como

um campo de conhecimento e de prática contínua e permanente, transdisciplinar, intersetorial e multiprofissional que visa promover a prática autônoma e voluntária de hábitos alimentares saudáveis. A prática da EAN deve fazer uso de abordagens e recursos educacionais problematizadores e ativos que favoreçam o diálogo junto a indivíduos e grupos populacionais, considerando todas as fases do curso da vida, etapas do sistema alimentar e as interações e significados que compõem o comportamento alimentar (BRASIL, 2012, p.23). 
Diante disso, a criação de hortas é uma proposta pedagógica comum no cotidiano das escolas. Contudo, vários estudos demonstram que pode ser reduzida a uma perspectiva produtivista que objetiva, essencialmente, os atos de plantar e colher para promoção da saúde humana.

$\mathrm{Na}$ pesquisa realizada neste estudo, foram encontrados 115 resultados nas duas bases descritas e considerando o período avaliado, sendo que 40 estudos foram descartados $(34,8 \%)$ por não apresentarem relação com a horta escolar. Os resultados obtidos nas bases de dados durante cinco anos (2014 2018), como pode-se observar na Tabela 2 (próxima página), apontam que, dos 75 estudos selecionados, 63 tratam a horta escolar por uma abordagem que se volta à perspectiva produtivista e reducionista. E somente 12 poderiam ser relacionados a aspectos mais abrangentes, como a construção de valores, de autonomia, oportunidade de interação social, sensibilização ambiental, conexão com a natureza, questões políticas e sociais das comunidades e reflexões sobre as emoções sentidas no manejo da horta escolar. Não foi verificada diferenciação entre as duas perspectivas - produtivista/reducionista e aspectos da ecologia profunda - entre os artigos mais antigos e os mais recentes. Esse fato pode demonstrar que ainda não ocorreram mudanças significativas nas formas de utilizar os variados potenciais pedagógicos da horta escolar.

Dentre os artigos classificados na visão da ecologia profunda, Huys et al. (2017) analisaram a percepção de professores e estudantes sobre a horta escolar. O objetivo inicial da implantação da metodologia educativa foi envolver os alunos com a natureza e não o de incentivar que ingerissem mais frutas e legumes, como foi relatado na maioria dos outros artigos selecionados. Priorizou-se a Educação Ambiental e não a melhoria da saúde. Os entrevistados não perceberam o aumento do consumo de frutas e legumes, pois os pais não estavam envolvidos e os estudantes não passaram tempo suficiente na horta escolar para analisar. Contudo, perceberam melhoria no conhecimento e atitudes dos alunos em relação às plantas cultivadas. A implantação da horta envolveu a participação da sociedade, por meio do financiamento de organizações, doações de pais e o lucro das vendas dos vegetais também foi convertido na manutenção do projeto. Os autores consideraram a horta como uma forma de aproximar a comunidade da escola, colaborando no cuidado e participando na doação de espaços para a sua ampliação. Geralmente, a falta de local se constitui como um dos principais entraves encontrados pelas instituições educativas. Além disso, os participantes do estudo relataram que tiveram dificuldades para associar 0 trabalho na horta com o currículo. Contudo, os autores apresentaram formas variadas de realizar essa integração e sugeriram relacionar a horta aos objetivos de desenvolvimento definidos pelo Ministério da Educação de Flanders, Bélgica. 
Tabela 2: Artigos selecionados nas bases pesquisadas entre os anos de 2014 e 2018. Os resultados duplicados nas duas bases pesquisadas foram colocados somente na coluna da base Scopus.

\begin{tabular}{|c|c|c|}
\hline ARTIGOS ANALISADOS & SCOPUS & WEB OF SCIENCE \\
\hline $\begin{array}{l}\text { Aspectos da perspectiva } \\
\text { reducionista/produtivista }\end{array}$ & 46 & 17 \\
\hline $\begin{array}{l}\text { Aspectos da perspectiva } \\
\text { Ecologia Profunda }\end{array}$ & 5 & 7 \\
\hline $\begin{array}{l}\text { Total de } \\
\begin{array}{l}\text { pesquisados } \\
\text { base }\end{array}\end{array}$ & 69 & 107 \\
\hline $\begin{array}{l}\text { Autores } \\
\text { Aspectos da perspectiva } \\
\text { reducionista/produtivista }\end{array}$ & $\begin{array}{l}\text { Taylor et al. (2018); Greer et al. } \\
\text { (2018); Loso et al. (2018); } \\
\text { Schreinemachers et al. (2017a); Nury } \\
\text { et al. (2017); Turner et al. (2017); } \\
\text { Schreinemachers et al. (2017b); } \\
\text { DeCosta et al. (2017); Scherr et al. } \\
\text { (2017); Savoie-Roskos et al. (2017); } \\
\text { Erismann et al. (2017); Laurie et al. } \\
\text { (2017); Beccarelli et al. (2017); Davis } \\
\text { \& Brann (2017); Turner et al. (2016); } \\
\text { Kararo et al. (2016); Evans et al. } \\
\text { (2016a); Evans et al. (2016 b); Ohly et } \\
\text { al. (2016); Erismann et al. (2016); Utter } \\
\text { et al. (2016); Monroy Díaz et al. } \\
\text { (2016); Lyons et al. (2015); Geller et } \\
\text { al. (2015); Spears-Lanoix et al. (2015); } \\
\text { Davis \& Somerset (2015); Wansink et } \\
\text { al. (2015); Hutchinson et al. (2015); } \\
\text { Martinez et al. (2015); Collins et al. } \\
\text { (2015); Jones et al. (2015); Berezowitz } \\
\text { et al. (2015); Triador et al. (2015); } \\
\text { Jackson (2015); Kupolati et al. (2015); } \\
\text { Marín Rives et al. (2015); Urey \& } \\
\text { Çepni . (2014); Christian et al. (2014); } \\
\text { Sedibe et al. (2014); Ganann et al. } \\
\text { (2014); Arko-Achemfour (2014); } \\
\text { Guitart et al. (2014); Eckermann et al. } \\
\text { (2014); Rangel et al. (2014); Yoder et } \\
\text { al. (2014); Slater Et al. (2014); }\end{array}$ & $\begin{array}{l}\text { Hume et al. (2014); } \\
\text { Dominguez (2015); } \\
\text { Duncan et al. (2015); } \\
\text { Hanbazaza et al. } \\
\text { (2015); Grier et al. } \\
\text { (2015); Linnell (2016); } \\
\text { Eno et al. (2016); } \\
\text { Wenhold \& Faber } \\
\text { (2016); Coelho \& } \\
\text { Bógus (2016); Costa et } \\
\text { al. (2016); Allirot et al. } \\
\text { (2016); Gresse et al. } \\
\text { (2017); Gatto et al. } \\
\text { (2017); Storz \& } \\
\text { Heymann (2017); Lee } \\
\text { et al. (2017); Sarti et al. } \\
\text { (2017); Mangadu et al. } \\
\text { (2017); }\end{array}$ \\
\hline $\begin{array}{l}\text { Autores } \\
\text { Aspectos da perspectiva } \\
\text { Ecologia Profunda }\end{array}$ & $\begin{array}{l}\text { Huys et al. (2017); Kangas et al. } \\
\text { (2017); Bonilla (2014); Petrou \& } \\
\text { Korfiatis (2016); Hellermann (2017); }\end{array}$ & $\begin{array}{l}\text { Leone et al. (2014); } \\
\text { Sanabria et al. (2015); } \\
\text { Tong \& Wang (2016); } \\
\text { Burt (2016); Chang et } \\
\text { al. (2016); Burt et al. } \\
\text { (2017); Delia \& Krasny } \\
\text { (2018) }\end{array}$ \\
\hline
\end{tabular}

Fonte: Dados da pesquisa.

Kangas et al. (2017) desenvolveram um estudo de caso no qual analisaram as formas de aprendizagem de uma específica aluna em diferentes contextos educacionais, a fim de verificar o processo de tomada de decisão e iniciativas dessa estudante. Os locais analisados foram a sala de aula, a horta escolar e uma fazenda próxima. A pesquisa demonstrou que em sala de aula a 
aluna esteve mais propensa a executar as instruções do professor e que a iniciativa ficou limitada a realizar perguntas e compartilhar as informações com os colegas. Em comparação com os outros ambientes de aprendizagem, a horta escolar foi o local em que a estudante apresentou o maior número de iniciativas. Percebeu-se que, nesse espaço, os alunos tinham maior liberdade na tomada de decisão.

Bonilla (2014) descreve, em seu estudo, o papel e as percepções das lideranças latinas na organização de uma horta na escola Walnutbrook Elementary, nos Estados Unidos. O pesquisador foi encarregado de incentivar as famílias mexicanas a se inserirem nas tomadas de decisões da comunidade escolar, pois a maior parte das deliberações na instituição era tomada por pessoas brancas. Verificou-se a luta das lideranças latinas para auxiliar na horta, no sentido de fazer com que a escola transmitisse os valores educacionais dessas famílias. A horta serviu como ferramenta para lutar contra as desigualdades raciais na unidade de ensino. A prática extrapolou os limites da escola e foi levada pelas mães participantes do projeto para as suas comunidades e círculos sociais.

Delia e Krasny (2018) realizaram um estudo sobre como fornecer recursos para o desenvolvimento da juventude no contexto da administração ambiental nas cidades. Jovens foram envolvidos em fazendas urbanas, hortas comunitárias e no mercado dos fazendeiros. Com isso, desenvolveram habilidades de gerenciamento de vendas de produtos, de cuidado e comunicação com outros jovens e com a comunidade em geral, assim como criaram consciência crítica da justiça no sistema alimentar, que diz respeito à noção do direito ao acesso a alimentos de qualidade, à segurança alimentar etc.

Sanabria et al. (2015), em seu estudo, salientam que as crianças envolvidas tiveram a visão de que a horta é importante para o aprendizado, para desenvolver 0 trabalho em equipe e adquirir conhecimento sobre o manejo da terra. Os alunos relataram que a venda das hortaliças auxiliou na renda das famílias, proporcionou momentos de divertimento e cuidado com a natureza, além de oportunizar a realização de projetos investigativos. As pesquisas realizadas pelos alunos da $5^{\mathrm{a}}$ série em torno da horta escolar foram utilizadas na construção de um site informativo, que contou com a participação dos alunos, professores e pais. Esse processo estimulou o desenvolvimento da liderança nos envolvidos e a socialização do conhecimento gerado.

Tong e Wang (2016) demonstraram que pátios verdes e jardins comestíveis resultam de um esforço altamente colaborativo entre escolas, comunidades e organizações. Esses espaços não apenas ampliam a sala de aula ao ar livre, mas também promovem a aprendizagem, treinamento físico, conscientização dos recursos alimentares e de alimentos frescos. As crianças atendidas no projeto obtiveram mais conhecimento sobre os processos cíclicos da natureza, sobre hábitos alimentares, sobre o cultivo do solo, relações entre o sol e a terra e desenvolveram habilidades artísticas e de escrita. 
Chang et al. (2016) realizaram uma pesquisa, em uma horta escolar, com 20 meninos e 23 meninas. Os resultados demonstraram que os alunos desenvolveram habilidades para a vida, como a autoestima, senso de responsabilidade e conquista, paciência, atenção, resiliência, iniciativa na resolução de problemas, desejo de trabalhar, compaixão e empatia. Eles tiveram sentimentos agradáveis ao cultivar os alimentos, melhoraram os relacionamentos, adquiriram novos conhecimentos, experimentaram o aroma e o sabor das frutas e legumes, fatores que melhoraram o cuidado com a saúde e aumentaram a conexão com a natureza.

Burt et al. (2017) pesquisaram que 16 componentes curriculares diferentes de 21 escolas de Nova York utilizavam a/o horta/jardim para ensinar. Os componentes de ciência, alimentação/nutrição, agricultura/cultivo de alimentos, artes da língua inglesa, matemática, ciências ambientais, história, saúde, economia doméstica, artes, serviço comunitário, estudos sociais, informática, educação física, língua estrangeira e artes cênicas ocupam o local para a aprendizagem. As atividades na horta escolar exigiram que os professores adquirissem novos conhecimentos e que ensinassem de formas diferentes os temas que conheciam previamente.

No trabalho de Leone et al. (2014) é relatado o processo de restauração ecológica e de horticultura em um quintal de uma escola abandonada na região do Rio Papireto, em Palermo, Itália. Avaliações ambientais e sociais equivocadas fizeram com que 0 rio secasse e ocorresse degradação socioambiental. Foi realizada uma escavação para que a água do rio ressurgisse e o solo retirado foi utilizado para encher caixas feitas com pallets, onde foram cultivadas algumas plantas. Por meio de um jogo, buscando o "equilíbrio de Nash", os participantes voluntários (idosos, donas de casa, alunos, desempregados, monges capuchinhos, entre outros), juntamente com especialistas, buscaram formas de recuperar a beleza do ambiente, ou seja, desenvolveram uma nova maneira de planejamento da paisagem urbana.

Petrou e Korfiatis (2016) relataram, em seu estudo, a utilização da "Teoria da atividade" para transformação dos indivíduos e do ambiente social e físico. Conforme os autores, a aprendizagem emerge das interações entre os seguintes elementos: os sujeitos (participantes), os objetos (horta), a comunidade (voluntários que auxiliaram os participantes), ferramentas (solo, enxadas, entre outros), as regras (cuidados com os vegetais) e a divisão do trabalho (papéis dos participantes e de outros membros da comunidade). 0 ambiente de aprendizagem criado envolveu atividades de observação, ação e reflexão, assim como descoberta, resolução de problemas e análise crítica. Demonstrou-se, também, como a comunidade escolar valoriza, interpreta e reflete sobre sua participação em um projeto de elaboração de horta escolar.

Hellermann (2017) relata, por meio de um estudo realizado com estudantes universitários, que a horta proporcionou bem-estar físico, emocional e espiritual, agilidade, confiança, conhecimentos práticos de jardinagem, provocando o senso de comunidade. Além disso, por meio das vivências 
proporcionou aprendizagem e momentos de reflexão, estimulou a formação de equipes e a resolução de problemas.

Para Burt (2016), as hortas escolares atenderam às necessidades sociais de vários contextos políticos ao longo do tempo. Esses espaços foram vistos de várias maneiras, como oportunidade de estudo da natureza, de combater a prevalência de doenças no ambiente urbano, demonstrar patriotismo, diminuir os efeitos da escassez de alimentos, promover a administração ambiental, propiciar a alfabetização ecológica, estimular o consumo de alimentos frescos e combater a obesidade. Porém, as funções de nutrir adequadamente as crianças e proporcionar um ambiente de aprendizado significativo e envolvente permaneceram relativamente estáveis. Para o autor, o sucesso desses espaços se deve à capacidade de atender às demandas sociais e políticas de cada época.

Por meio do trabalho com as hortas escolares pode-se despertar a autonomia dos indivíduos, a alfabetização científica, estimular reflexões sobre a segurança alimentar, desenvolver habilidades artísticas, matemáticas, linguísticas etc. e ensinar uma profissão. É possível sensibilizar para a sustentabilidade, despertar os sentidos, estabelecer a conexão com a mãeTerra e com os processos que sustentam e mantêm a vida, fortalecendo as relações de cuidado e respeito com o meio, consigo e com os demais seres. Conforme Capra (2006), essa metodologia pode religar as crianças à essência da vida e, concomitantemente, integrar e enriquecer praticamente todas as atividades escolares. A horta pode facilitar o entendimento científico por meio da compreensão dos fenômenos cíclicos naturais, do fluxo de energia nas cadeias e teias alimentares, da fertilidade do solo e da sua relação com a manutenção da vida. Como consequência, os indivíduos podem se engajar de maneira individual ou coletiva nas causas ambientais, sendo agentes promotores de mudanças. É uma metodologia de ensino que pode promover a sustentabilidade.

Ao ser integrado no currículo escolar, o trabalho com a horta pode fornecer informações sobre segurança alimentar e estimular mudanças na cultura alimentar. Para Capra (2006), esse espaço é uma sala de aula que auxilia no sentido de religar os envolvidos aos fundamentos básicos da comida, à essência da vida.

Quando a horta da escola passa a fazer parte do currículo, nós aprendemos sobre os ciclos alimentares, por exemplo, e integramos os ciclos alimentares naturais aos ciclos do plantio, cultivo, colheita, compostagem e reciclagem. Por meio dessa prática, descobrimos inclusive que a horta da escola, em sua totalidade, está embutida em sistemas maiores que também são teias vivas com seus próprios ciclos. Os ciclos alimentares se cruzam com estes ciclos maiores - o ciclo da água, o ciclo das estações, e assim por diante -, todos eles formando conexões na teia da vida planetária (CAPRA, 2006, p.14-15). 
Segundo a professora Alice Waters do projeto chamado "Pátio Escolar Comestível", a horta não pode ser uma ferramenta isolada, isto é, sem relação com o refeitório e a cozinha (WATERS, 1999). Tudo deve estar interligado a um currículo ecológico (STONE, 2006). O referido projeto foi realizado na escola de Ensino Médio Martin Luther King, nos Estados Unidos, e instigou aos educadores a pensar em novas formas de utilizar a horta no cotidiano escolar.

As crianças merecem ser educadas em lugares dos quais possam sentir orgulho. [...] O objetivo da educação é o de prover as crianças com um sentido de finalidade e um sentido de possibilidade, com habilidades e hábitos de pensar que vão ajudá-los a viver no mundo. Uma forma chave de viver estas habilidades e hábitos é aprender como comer bem e como comer direito. Um currículo criado para educar os sentidos e a consciência - um currículo baseado em agricultura sustentável vai ensinar às crianças a sua obrigação moral de serem vigias e comissárias dos recursos finitos do nosso planeta e ensinará a elas a alegria da mesa, os prazeres do trabalho real e o real significado de comunidade (WATERS, 1999, p. 13).

Para Waters (1999), aprende-se melhor a valorizar e respeitar um ao outro estando à mesa. As refeições das famílias tornaram-se mais raras atualmente. Por isso, a escola deve pensar o que pode fazer em relação a isso. As escolas ensinam os estudantes sem levar em consideração as emergências familiares de um lado e a emergência planetária do outro.

A Educação Ambiental, por meio da vivência, reconhece os indivíduos de maneira integral, incluindo e priorizando o aprendizado por meio do corpo, dos sentidos e da percepção mais sutil de si mesmo, dos outros, do mundo, da natureza e dos processos que originam e mantêm a vida. $A$ integração do corpo nas metodologias pedagógicas experimentais trata o corpo como um elemento pertinente à aprendizagem (MENDONÇA, 2007, p. 119).

A experiência humana tem sido moldada e enriquecida por viver com a beleza e a complexidade do mundo natural. [...] Hoje em dia, brincar significa brinquedos plásticos, dispositivos eletrônicos e é, geralmente, uma experiência solitária. No Pátio Comestível da Escola, os estudantes têm uma chance, extremamente necessária, de experimentar, em primeira mão, a areia, barro, lama, humus, adubo, água, ar, vento, chuva, luz solar, insetos, aranhas, plantas, raízes, folhas e ramos (HAWKINS, 1999, p. 19).

É necessário abandonar os preconceitos e as relações de dominação entre professor e aluno sob o intuito de educar para a sustentabilidade e 
reconhecer os indivíduos como seres em processo de formação, que constroem e desconstroem o conhecimento e 0 mundo de forma compartilhada.

Conforme Leff (2002, p. 219),

a educação deve preparar as novas gerações não apenas para que aceitem a incerteza (uma educação como preparação face ao desastre ecológico e como criação de capacidades de resposta diante do imprevisto); ela deve preparar também novas mentalidades, capazes de compreender as complexas interrelações entre os processos objetivos e subjetivos que constituem seus mundos de vida, para gerar habilidades inovadoras tendo em vista a construção do inédito. Trata-se de uma educação que permite que os indivíduos se preparem para a construção de uma nova racionalidade; não para uma cultura de desesperança e alienação, mas pelo contrário, para um processo de emancipação que permita o surgimento de novas formas de reapropriação do mundo.

Conforme Pelegrini (2006), o indivíduo, ao se reconhecer como parte integrante do seu entorno, pode aumentar a autoestima e valorizar a sua identidade cultural, engajando-se em relação à preservação do patrimônio em toda a sua abrangência. $O$ conhecimento interiorizado e a apropriação dos bens culturais pela sociedade são essenciais, pois fortalecem os sentimentos de identidade e pertencimento, assim como podem estimular a luta pelos direitos dos sujeitos e o exercício da cidadania.

O reconhecimento da interdependência entre os seres e do valor intrínseco da natureza pode despertar, nos indivíduos, a consciência do seu papel na teia da vida e, com isso, promover a autonomia e o sentimento de responsabilidade para com o cosmos.

\section{Conclusões}

Desde a promulgação da Política Nacional de Educação Ambiental (PNEA), estabelecida pela Lei no 9.795, a escola e a Educação Ambiental ganham centralidade na constituição da consciência ambiental no Brasil. Assim, é papel da instituição escolar promover atividades e fomentar valores que visem educar para a sustentabilidade. Contudo, como a análise das pesquisas apontou, faz-se necessário minimizar as percepções antropocêntricas para o enfrentamento da crise ambiental e educar dentro dos novos paradigmas ecológicos, para que uma visão de mundo mais voltada ao biocentrismo seja promovida.

A horta escolar pode ser uma importante metodologia educativa no sentido de despertar para a ecologia profunda e para a sustentabilidade. 
Contudo, a maioria dos estudos publicados ainda apontam que o trabalho com a horta está sujeito a uma visão reducionista e produtivista. No ato de cultivar o solo, pode-se proporcionar a sensibilização ambiental, o reconhecimento do valor intrínseco da natureza, promover discussões sobre temas mais profundos e filosóficos com o intuito de que a humanidade se perceba como parte da "teia da vida". Entretanto, ainda são poucos os estudos publicados que mencionam a horta escolar, na perspectiva da ecologia profunda, como instrumento metodológico educativo. O que demonstra a necessidade de promoção de práticas pautadas pela sensibilização ambiental a partir dessa abordagem educativa, pois, com isso, auxiliaria a resgatar elementos muito mais proveitosos para os objetivos ambientais nacionais. Ademais, é pertinente insistir e investir em esforços que resultem na publicação dessas ações, compartilhando conhecimentos e proporcionando avanço teórico.

Por fim, as conclusões desta pesquisa apontam para a promoção de duas dimensões extremamente importantes. A primeira é a educativa, diz respeito à prática pedagógica necessária no espaço escolar, que precisa recuperar ações voltadas à ecologia profunda. A segunda diz respeito às políticas públicas educacionais, que carecem de estímulos de programas cujo foco deveria ser realizar ações experimentais nas escolas, através de financiamento e editais, com o objetivo de estimular ações interdisciplinares e projetos de diferentes matérias, garantindo, assim, práticas que atravessam os currículos e estimulam ações voltadas à ecologia profunda nos espaços escolares.

\section{Referências}

ALLIROT, X. et al. Involving children in cooking activities: A potential strategy for directing food choices toward novel foods containing vegetables. Appetite. v. 103, p. 275-285, 2016.

ARKO-ACHEMFUOR, A. Teaching the youth and adults through education with production: The case study of Tiger Kloof Educational Institution. Mediterranean Journal of Social Sciences MCSER Publishing. v. 5, n.1, 2014.

BECCARELLI, L. M. et al. Using Skin Carotenoids to Assess Dietary Changes in Students After 1 Academic Year of Participating in the Shaping Healthy Choices Program. Journal of Nutrition Education Behavior. v. 49, i. 1, p.7378, 2017.

BEREZOWITZ, C. K. et al. School Gardens Enhance Academic Performance and Dietary Outcomes in Children. Journal of School Health, v. 85, i. 8, p. 508-518, 2015.

BONILLA, C. M. Racial counter narratives and Latina epistemologies in relational organizing. Anthropology e education quarterly. v. 45, i. 4, p. 391404, 2014.

revista brasileira educação ambiental 
BRASIL. Lei no 9.795, de 27 de abril de 1999. Dispõe sobre a Educação Ambiental, institui a Política Nacional de Educação Ambiental e dá outras providências. Política Nacional de Educação Ambiental. Brasília, DF.

BRASIL. Decreto 4.281, de 25 de junho de 2002. Regulamenta a Lei 9.795/1999, que institui a Política Nacional de Educação Ambiental e dá outras providências. Disponível em: $<$ http://www.planalto.gov.br/ccivil 03/decreto/2002/D4281.htm> Acesso em: 15/03/2021.

BRASIL. Lei 11.346, de 15 de setembro de 2006. Cria o Sistema Nacional de Segurança Alimentar e Nutricional. SISAN com vistas em assegurar o direito humano à alimentação adequada e dá outras providências. Sistema Nacional de Segurança Alimentar e Nutricional. Brasília, DF.

BRASIL. Ministério do Desenvolvimento Social e Combate à Fome. Marco de referência de educação alimentar e nutricional para as políticas públicas. Brasília, DF, 2012.

BURT, K. G. A Complete History of the Social, Health, and Political Context of the School Gardening Movement in the United States: 1840-2014. Journal of Hunger \& Environmental Nutrition. v. 11, i. 3, 2016.

BURT, K. G. et al. Development of the GREEN (Garden Resources, Education, and Environment Nexus) Tool: An Evidence-Based Model for School Garden Integration. Journal of the Academy of Nutrition and Dietetics. v. 117, i. 10, p. 1517-1527, 2017.

CAPRA, F. A Teia da Vida. São Paulo: Editora Cultrix, 1996.

CAPRA, F. Como a natureza sustenta a teia da vida. In: STONE, M. K.; BARLOW, Z. (Org.) Alfabetização ecológica: a educação das crianças para um mundo sustentável. São Paulo: Cultrix. 2006. p. 14.

CEBALLOS, G. et al. Accelerated modern human-induced species losses: Entering the sixth mass extinction. Sciences Advances. Vol. 1. №.5, 2015.

CHANG, Y. Y. et al. Exploring the Benefits of School Gardening for Children in Taiwan and Identifying the Factors Influencing these Benefits. American Society for Horticultural Science. v. 26, i. 6, 2016.

CHRISTIAN, M. S. et al. Evaluation of the impact of a school gardening intervention on children's fruit and vegetable intake: A randomised controlled trial. International Journal of Behavioral Nutrition and Physical Activity. v. 11, i. 99, 2014.

COELHO, D. E. P.; BÓGUS, C. M. Vivências de plantar e comer: a horta escolar como prática educative, sob a perspectiva dos educadores. Saúde e Sociedade. São Paulo: v. 25, n. 3, 2016.

COSTA, R. et al. Environmental education through community horta: study in a public school city of São Paulo. Revista Científica Hermes. v. 16, 2016. 
COLLINS, C. et al. Food for thought: Edible gardens in New Zealand primary and secondary schools. Health Promotion Journal of Australia. v. 26, i. 1, p. 70-73, 2015.

DAVIS, K. L.; BRANN, L. S. Examining the Benefits and Barriers of Instructional Gardening Programs to Increase Fruit and Vegetable Intake among PreschoolAge Children. Journal of Environmental and Public Health. 2017.

DAVIS, J. N.; SOMERSET, S. Sustenance and sustainability: Maximizing the impact of school gardens on health outcomes. Public Health Nutrition. v. 18, i. 13, p. 2358-2367, 2015.

DECOSTA, P. et al. Changing children's eating behaviour - A review of experimental research. Appetite. v. 113, p. 327-357, 2017.

DELIA, J. KRASNY, M. E. Cultivating Positive Youth Development, Critical Consciousness, and Authentic Care in Urban Environmental Education. Frontiers in Psychology. v.8, 2018.

DOMINGUEZ, P. R. 2015. Impact of School-Based Interventions on Children's Healthful Nutritional Outcomes (2009-2013): Implications for Future Research. Journal of Child Health and Nutrition. p. 180-193, 2015.

DUNCAN, M. J. et al. The impact of a school-based gardening intervention on intentions and behaviour related to fruit and vegetable consumption in children. Journal of Health Psychology. v. 20, i. 6, p. 765-773, 2015.

ECKERMANN, S. et al. Evaluating return on investment in a school based health promotion and prevention program: The investment multiplier for the Stephanie Alexander Kitchen Garden National Program. Social Science \& Medicine, v. 114, p. 103-112, 2014.

ENO, E. G. J. et al. Horta na escola: incentivo ao cultivo e a interação com o meio ambiente._Revista Eletrônica em Gestão, Educação e Tecnologia Ambiental. Santa Maria: v. 19, n. 1, p. 248-253, 2016.

ERISMANN, S. et al. Complementary school garden, nutrition, water, sanitation and hygiene interventions to improve children's nutrition and health status in Burkina Faso and Nepal: A study protocol. BMC Public Health. v. 9, i. 16, 244, 2016.

ERISMANN, S. et al. Prevalence and risk factors of undernutrition among schoolchildren in the Plateau Central and Centre-Ouest regions of Burkina Faso Infectious Diseases of Poverty. v. 6, i. 17, 2017.

EVANS, A. et al. Previous Gardening Experience and Gardening Enjoyment Is Related to Vegetable Preferences and Consumption Among Low-Income Elementary School Children. Journal of Nutrition Education Behavior. v. 48, i.9, p. 618-624, 2016a.

EVANS, A. et al. Impact of school-based vegetable garden and physical activity coordinated health interventions on weight status and weight-related behaviors of ethnically diverse, low-income students: Study design and baseline data of 
the Texas, Grow! Eat! Go! (TGEG) cluster-randomized controlled trial. BMC Public Health. v. 13, i. 16, 973, 2016 b.

GANANN, R. et al. Enhancing nutritional environments through access to fruit and vegetables in schools and homes among children and youth: A systematic review. BMC Research Notes. v. 4, i. 7, 422, 2014.

GATTO, N. M. et al. LA sprouts randomized controlled nutrition, cooking and gardening programme reduces obesity and metabolic risk in Hispanic/Latino youth. Pediatric Obesity. v.12, i. 1, 2017, p. 28-37.

GELLER, K. S. et al. Measuring elementary-aged children's self- efficacy and proxy efficacy for gardening and related health behaviors. HortTechnology. v. 25, i. 6, 731-741, 2015.

GOLDIM, J. R. Bioética: conceitos fundamentais: bioética: definição de bioética - Potter 1998: ecologia profunda. Porto Alegre, 1999. Disponível em: $<$ https://www.ufrgs.br/bioetica/ecoprof.htm>. Acesso em: 07/10/2017

GRESSE, A. et al. Situational analysis: implementation of the national school Nutrition Programme in low socio-economic primary schools in Nelson Mandela Bay. Journal of Consumer Sciences. v. 2, 2017.

GREER, A. E. et al. Agricultural Experiences Are Positively Associated With High School Students' Fruit and Vegetable Perceptions and Consumption. Journal of Nutrition Education Behavior. v. 50, i. 2, p. 133-140, 2018.

GREGORY, A.T.; DENNISS, A.R. An Introduction to Writing Narrative and Systematic Reviews - Tasks, Tips and Traps for Aspiring Authors. Heart, Lung And Circulation, [s.I.], v. 27, n. 7, p.893-898, jul. 2018.

GRIER, K. et al. Feasibility of an experiential community garden and nutrition programme for youth living in public housing. Public Health Nutrition. v. 18, s. 15, p. 2759-2769, 2015.

GUITART, D. A. et al. Color me healthy: Food diversity in school community gardens in two rapidly urbanising australian cities. Health Place. v. 26, p. 110117, 2014.

GRÜN, M. Ética e Educação Ambiental: a conexão necessária. 6. ed. Campinas: Papirus, 2002.

GRÜN, M. O conceito de holismo em ética ambiental e em Educação Ambiental. In: SATO, M. e CARVALHO, I. (Organizadores). Educação Ambiental: Pesquisa e Desafios. Porto Alegre. Ed. Artmed, 2005. p. $43-50$.

HANBAZAZA, M. et al. The Impact of School Gardening on Cree Children's Knowledge and Attitudes toward Vegetables and Fruit. Canadian Journal of Dietetic Practice and Research. v. 76, n.3, 2015.

HAWKINS, D. A experiência da horta. In: CAPRA, F. et al. 0 pátio comestível da escola. Califórnia: Centro para Ecoalfabetização, 1999. p. 19-25. 
HELLERMANN, M. Gardening on the Fringes: A Case Study at City Tech. Food, Culture \& Society - An International Journal of Multidisciplinary Research. v. 20, i. 4, 2017.

HUME, A. et al. Remote school gardens: exploring a cost-effective and novel way to engage Australian Indigenous students in nutrition and health. Australian and New Zealand Public Journal of Health. v. 38, n. 3, p. 235240, 2014.

HUTCHINSON, J. et al. Evaluation of the impact of school gardening interventions on children's knowledge of and attitudes towards fruit and vegetables. A cluster randomised controlled trial. Appetite. v. 91, p. 405-414, 2015.

HUYS, N. et al. School Gardens: A Qualitative Study on Implementation Practices. International Journal of Environmental Research and Public Health. v. 14, i. 12, 2017.

JACKSON, E. Connecting people to the source of their food: changing the way we think about food to improve health. North Carolina Medical Journal. v. 76, i. 1, p. 54-56, 2015.

JONES, S. J. et al. SC Farm-to-School Programs Encourages Children to Consume Vegetables. Journal of Hunger \& Environmental Nutrition. v. 10, i. 4, p. 511-525, 2015.

KANGAS, M. et al. 'I'll take care of the flowers!' Researching agency through initiatives across different learning environments. Journal of Adventure Education and Outdoor Learning. v. 17, 2017.

KARARO, M. J. et al. Eat your way to better health: Evaluating a garden-based nutrition program for youth. HortTechnology. v. 26 n. 5, p. 663-668, 2016.

KUPOLATI, M. D. et al. Teachers' perceptions of school nutrition education's influence on eating behaviours of learners in the Bronkhorstspruit District. South African Journal of Education. v. 35, n. 2, p. 1-10, 2015.

LAURIE, S. M. Assessment of food gardens as nutrition tool in primary schools in South Africa. South African Journal of Clinical Nutrition. v. 30, i. 4, 2017.

LEE, R. E. et al. Sustainability via Active Garden Education (SAGE): results from two feasibility pilot studies. BMC Public Health. v. 17, i. 242, 2017.

LEFF, E. Epistemologia Ambiental. 5. ed. São Paulo, Ed. Cortez, 2002.

LEONE, M. S. et al. Danisinni "Laughes" and Plants Papyrus in Exhumed Papireto River. Proceedings of the 18th IPSAPA/ISPALEM International Scientific Conference. Catania (Italy). 2014.

LINNELL, J. D. Using a Systematic Approach and Theoretical Framework to Design a Curriculum for the Shaping Healthy Choices Program. Journal of Nutrition Education and Behavior. v. 48, i. 1, p. 60-69, 2016. 
LOSO, J. et al. Gardening Experience Is Associated with Increased Fruit and Vegetable Intake among First-Year College Students: A Cross-Sectional Examination. Journal of the Academy of Nutrition and Dietetics. v. 118, i. 2, p. 275-283, 2018.

LOVATTO, P. B. et al. Ecologia profunda: o despertar para uma Educação Ambiental complexa. Redes, Santa Cruz do Sul, v. 16, n. 3, p. 122 - 137, set/dez 2011.

LYONS, G. H. Promoting nutritious leafy vegetables in the Pacific and northern Australia. Acta Horticulturae. v. 1102, p. 253-260, 2015.

MALHOTRA, N. K. Pesquisa de Marketing. Uma orientação aplicada. Tradução de Lene Belon Ribeiro, Monica Stefani. 6. ed. Porto Alegre: Bookman, 2012.

MANGADU, T. et al. Best practices for community gardening in a US-Mexico border community. Health Promotion International. v. 32, i. 6, 2017.

MARÍN RIVES, F. et al. Atención farmacéutica en el fomento del desayuno saludable desde la oficina de farmacia. Nutrición Hospitalaria. v. 32, n.3, 2015.

MARIOTTI, H. Prefácio. In: MATURANA, H. R.; VARELA, F. J. A árvore do conhecimento - As bases biológicas da compreensão humana. São Paulo: Ed. Palas Athena, 2001.

MARTINEZ, L. C. et al. Design and methodology of the LA Sprouts nutrition, cooking and gardening program for Latino youth: A randomized controlled intervention. Contemporary Clinical Trials. v. 42, p. 219-227, 2015.

MENDONÇA, R. Educação Ambiental vivencial. In: FERRARO JR., L.A. (Org.) Encontros e caminhos: Formação de educadoras (es) ambientais e coletivos educadores. Brasília: MMA, Departamento de Educação Ambiental, v. 2, p.119129, 2007

MONROY DÍAZ, G. et al. Características antropométricas y hábitos dietéticos en niños de etapa escolar del centro de salud con servicios ampliados de Tlalixtac de Cabrera, Oaxaca, México. Nutrición Clinica y Dietetica Hospitalaria. v. 36, i.3, p. 189-193, 2016.

NURY, E. et al. Sowing Seeds for Healthier Diets: Children's Perspectives on School Gardening. International Journal Environmental Research Public Health. v. 4, i. 7, 688, 2017.

OHLY, H. et al. A systematic review of the health and well-being impacts of school gardening: Synthesis of quantitative and qualitative evidence. BMC Public Health. v. 16, 286, 2016.

PELEGRINI, S. C. A. Cultura e natureza: os desafios das práticas preservacionistas na esfera do patrimônio cultural e ambiental. Revista Brasileira de História, São Paulo, v. 26, n. 51, p.115-140, 2006. 
PETROU, S.; KORFIATIS, K. J. School Kitchen-Gardens as Innovative Learning Environments for Supporting Environmental Education Pedagogies: Opportunities and Challenges for Teachers, Students and the Community. In: WALLACE, K. (ed.) Learning Environments: emerging theories. Applications and Future Directions. New York: NOVA Science Publishers, 2016. p. 51-72. 2016.

RANGEL, C. N. et al. Teaching and learning about food and nutrition through science education in Brazilian schools: An intersection of knowledge. Ciência \& Saúde Coletiva. v.19, n.9, 2014.

SANABRIA, D. et al. Web site as a strategy in the education for sustainability. Praxis \& Saber. v. 6, n. 11, p. 115-138, 2015.

SARTI, A. et al. "I Eat the Vegetables because I Have Grown them with My Own Hands': Children's Perspectives on School Gardening and Vegetable Consumption. Children \& Society. v. 31, i. 6, 2017.

SAVOIE-ROSKOS, M. R. et al. Increasing Fruit and Vegetable Intake among Children and Youth through Gardening-Based Interventions: A Systematic Review. Journal of the Academy of Nutrition and Dietetics. v. 117, i. 2, 2017, p. 240-250.

SAUVÉ, L. Educação Ambiental: possibilidades e limitações. Educação e Pesquisa, São Paulo, v. 31, n. 2, p. 317-322, maio/ago. 2005.

SCHERR, R. E. et al. A Multicomponent, School-Based Intervention, the Shaping Healthy Choices Program, Improves Nutrition-Related Outcomes. Journal of Nutrition Education Behavior. v. 49, i. 5, p. 368-379, 2017.

SCHREINEMACHERS, P. et al. School gardening in Bhutan: Evaluating outcomes and impact. Food Sec. v. 9, p. 635-648, 2017.

SCHREINEMACHERS, P. et al. Impact of school gardens in Nepal: a cluster randomised controlled trial. Journal of Development Effectiveness. v. 9, i. 3, p. 329-343, 2017.

SEDIBE, H. M. et al. Qualitative study exploring healthy eating practices and physical activity among adolescent girls in rural South Africa. BMC Pediatr. v. 26, i. 14, 211, 2014.

SLATER, E. et al. Health in Action Program, Brazil: A Program Impact Pathways (PIP) analysis. Food and Nutrition Bulletin. v. 35, i. 3, p. 108-116, 2014.

SORIA, E. C. R. Ecologia Humana e Ecologia Profunda na Práxis de Educação Ambiental da Escola da Natureza. Dissertação de Mestrado. Brasília, DF. 2012.

SPEARS-LANOIX, E. C. et al. Using Family-Focused Garden, Nutrition, and Physical Activity Programs to Reduce Childhood Obesity: The Texas! Go! Eat! Grow! Pilot Study. Childhood Obesity. v. 11, n. 6, 2015. 
STONE, M. K. Sustentabilidade: um novo prato no cardápio do almoço. In: STONE, M. K.; BARLOW, Z. (Org.) Alfabetização ecológica: a educação das crianças para um mundo sustentável. São Paulo: Cultrix. 2006. p.263-275.

STORZ, M.A.; HEYMANN, E.P. School Gardening: A Different Approach to Tackle Childhood Obesity? University of Toronto Medical Journal. v. 94, v. 2, 2017.

TAYLOR J. C. et al. Impact of a multicomponent, school-based nutrition intervention on students' lunchtime fruit and vegetable availability and intake: A pilot study evaluating the Shaping Healthy Choices Program. Journal of Hunger \& Environmental Nutrition. v. 13, i. 3, p. 415-428, 2018.

TONG, P.; WANG, S. Landscape Serving Education-Inspirations from Green School Yard in San Francisco. 5th International Conference on Materials Engineering for Advanced Technologies. p. 88-92, 2016.

TRIADOR, L. et al. A School Gardening and Healthy Snack Program Increased Aboriginal First Nations Children's Preferences Toward Vegetables and Fruit. Journal of Nutrition Education and Behavior. v. 47, i. 2, p. 176-180, 2015.

TURNER, L. et al. Increasing Prevalence of US Elementary School Gardens, but Disparities Reduce Opportunities for Disadvantaged Students. Journal of School Health. v. 86, i. 12, p. 906-912, 2016.

TURNER, L. et al. Facilitating Fresh: State Laws Supporting School Gardens Are Associated With Use of Garden-Grown Produce in School Nutrition Services Programs. Journal of Nutrition Education Behavior. v. 49, i. 6, p.481-489, 2017.

UREY, M.; CSEPNI, S. Evaluation of the effect of science-based and interdisciplinary school garden program on some science and technology course from different variables. Int J Behav Nutr Phys Act. v. 11, 99, 2014.

UTTER, J. et al. School gardens and adolescent nutrition and BMI: Results from a national, multilevel study. Preventive Medicine. v. 83, p. 1-4, 2016.

YODER, A. B. et al. Farm to Elementary School Programming Increases Access to Fruits and Vegetables and Increases Their Consumption Among Those With Low Intake. Journal of Nutrition and Education Behavior. v. 46, i. 5, p. 341-349, 2014

WANSINK, B. et al. A plant to plate pilot: A cold-climate high school garden increased vegetable selection but also waste. Acta Paediatrica. v. 104, i. 8, p. 823-826, 2015.

WATERS, A. Um mundo de possibilidades. In: CAPRA, F. et al. 0 pátio comestível da escola. Califórnia: Centro para Ecoalfabetização, p. 11-18, 1999.

WENHOLD, F. et al. Vitamin A-related potential of wild edible plants in a school vegetable garden: a case study from North-West Province, South Africa. Journal of Family Ecology and Consumer Sciences. Special Edition Food and nutrition challenges in Southern Africa, v. 1, 2016.

Revbea, São Paulo, V. 16, № 3: 79-100, 2021. 